\title{
Acinetobacter spp in a Third World Country with Socio-economic and Immigrants Challenges
}

\author{
Iman Dandachi ${ }^{1}$, Eid Azar ${ }^{1}$, Ramzi Hamouch ${ }^{1}$, Peter Maliha1 ${ }^{1}$, Samah Abdallah ${ }^{1}$, Elie Kanaan ${ }^{1}$, Rebecca \\ Badawi $^{1}$, Tamara Khairallah'1 ${ }^{1}$ Ghassan M Matar ${ }^{2}$, Ziad Daoud ${ }^{1,3}$ \\ ${ }^{1}$ Faculty of Medicine and Medical Sciences, Clinical Microbiology Laboratory, University of Balamand, Beirut, \\ Lebanon \\ 2 Department of Experimental Pathology, Immunology and Microbiology, American University of Beirut, Beirut, \\ Lebanon \\ ${ }^{3}$ Division of Clinical Microbiology, Saint George Hospital University Medical Center, Beirut, Lebanon
}

\begin{abstract}
Introduction: In the last decade, Acinetobacter species have taken a major public health concern. This is mainly due the increased resistance to a wide range of antibiotics causing treatment challenges. In view of the constant population mobilization and the economic crisis that Lebanon is currently facing, it becomes a necessity to re-evaluate the real threat of Acinetobacter spp and its implication in the one health.

Methodology: This review was conducted through the analysis of 45 research papers and reports pertaining to Acinetobacter spp performed in Lebanon. More than $82 \%$ of the papers consulted were published in international journals and more than 70 percent of them had received impact factor.

Results: An in depth description of the involvement of this organism in human infection and its role as potential pathogen or simple colonizer was performed. In addition, the different aspects of resistance, mostly to carbapenems and colistin was studied and summarized. While in animals and environment, susceptible strains were mostly isolated, OXA-23/OXA-24 were predominant in humans. Recently, NDM-1 producing Acinetobacter spp was detected in a Syrian refugee which then was reported in Lebanese patients. The bacterial identification procedures are non-systematic and not always reliable in the Lebanese studies presenting sometimes discrepancies an inconsistency.

Conclusion: Acinetobacter is commonly isolated Lebanon. In view of the spread of resistance among these isolated and their dissemination, Infection control measures attempting to control the spread of this genus in and outside hospitals are lacking and thus require more attention and stewardship activities.
\end{abstract}

Key words: Acinetobacter; bacterial resistance; carbapenems; oxacillinases; epidemiology.

J Infect Dev Ctries 2019; 13(11):948-955. doi:10.3855/jidc.11341

(Received 11 February 2019 - Accepted 08 March 2019)

Copyright (C) 2019 Dandachi et al. This is an open-access article distributed under the Creative Commons Attribution License, which permits unrestricted use, distribution, and reproduction in any medium, provided the original work is properly cited.

\section{Introduction}

In the last decades, the dissemination of antimicrobial resistance in Gram-negative bacilli (GNB) has caused major public health challenges worldwide [1]. Among others, Acinetobacter species are of special interest. This organism constitutes a complex saprophytic genus comprising a group of nonfermentative, facultatively anaerobic, Gram-negative coccobacilli. Acinetobacter spp are ubiquitous in nature being widely isolated from soil, wastewater, vegetables as well as humans and animals' skin [2]. The wide distribution of Acinetobacter species in different environments is mostly due to their ability to withstand desiccation, starvation and to survive on inanimate surfaces [3]. The Acinetobacter genus involves pathogenic and non-pathogenic species with Acinetobacter baumannii being the most common one implicated in hospital acquired infections especially in immunocompromised patients and to a lesser extent in community acquired ones [4]. Genotypically speaking, international clones I and II dominate the nosocomial outbreaks caused by this species worldwide [5]. Besides $A$. baumannii, there are increasing reports worldwide, highlighting the clinical relevance of other species such as Acinetobacter pitii, Acinetobacter nosocomialis and Acinetobacter calcoaceticus [4]. These species are difficult to be differentiated using the classical biochemical identification methods [6]. Species of Acinetobacter are known for their natural resistance to a wide range of clinically important antibiotics including $1^{\text {st }}$ and $2^{\text {nd }}$ generation cephalosporins and ertapenem [6]. Furthermore, the acquired resistance in these species is complex and involves an inter-play of several mechanisms: a) production of wide spectrum 
beta lactamases, notably class $\mathrm{D}$ oxacillinases and to a lesser extent class A and B carbapenemases, b) reduced expression of outer membrane proteins such as $\mathrm{CarO}$ and OprD and c) over-expression of efflux pumps including AdeABC belonging to the resistance nodulation cell division family; this latter mediates resistance toward beta-lactam and non-beta-lactam antibiotics including fluoroquinolones, aminoglycosides, erythromycin and chloramphenicol [2]. More recently, colistin resistance has also emerged in A. baumannii complicating thus further the clinical situation [7]. Acinetobacter spp is increasingly implicated in veterinary medicine as an emerging pathogen; cases have been reported in Switzerland and Germany [8]. Asymptomatic carriage of A. baumannii was also reported in livestock, companion animals, lice and arthropods [4]. Other clinically important species including $A$. pittii, A. nosocomialis, A. calcoaceticus and Acinetobacter lwoffii were detected in vegetables and dairy products [9].

In Lebanon, studies on Gram negative bacilli (GNB) have shown the wide production of beta lactamases [10]. One study conducted in the north of Lebanon, has shown that in 2009-2012, 28\% and 9\% of bacteremia episodes in neutropenic patients were caused by $3^{\text {rd }}$ generation cephalosporins and carbapenem resistant GNB respectively [1]. More recently, a countryside study conducted in 11 Lebanese hospitals revealed $1.2 \%, 41 \%$ and $88 \%$ of Enterobacteriaceae, Pseudomonas spp and $A$. baumannii respectively, were carbapenem nonsusceptible [10]. Enterobacteriaceae isolates harbored mainly the OXA-48 gene, whereas VIM-2, IMP-2 and IMP-1 were more prevalent in Pseudomonas spp. Among A. baumannii strains, OXA-23 and GES-11 were most commonly detected [10]. A recent nationwide study done by the Lebanese Society of Infectious Diseases (LSID) has shown that the rate of imipenem resistance in Acinetobacter species have significantly increased from $57.6 \%$ in 2011 to $84.5 \%$ in 2013 [11]. The identification of Acinetobacter species in most of these studies is done using standard microbiological techniques [12]. While in the clinical reports, A. baumannii was the most commonly identified specie; in environmental studies that mostly used molecular techniques a wider variety of Acinetobacter species was reported [8]. As Acinetobacter spp are generally known to originate from the environment, their identification is not as simple as it looks to be. Standard microbiological phenotypic as well as biochemical techniques are not highly reliable in view of the complexity of this genus
[6]. This puts a question mark on the accuracy of the numbers and percentages of Acinetobacter reported as A. baumannii. Subsequently, a false impression about the real occurrence of this specie might be given. In view of the recent demographic and socio-economic challenges imposed on the middle eastern region over the last 10 years, it is expected that the epidemiology of resistant Acinetobacter species would suffer significant changes under the pressure of continuous population mobilization and immigration. We attempt in this review to assess the real threat posed by Acinetobacter species and its implications on the clinical setting as well as in animals and environment in Lebanon; a Middle Eastern country with variable degrees of healthcare services and a significant burden of refugees.

\section{Acinetobacter baumannii in Lebanon: a real threat or an over-estimated colonizer?}

Acinetobacter species has been known since the early 1980 s to be among the most common Gramnegative bacilli causing nosocomial infections in Lebanon [13]. In 2004, multi-drug resistant (MDR) $A$. baumannii became a common isolate detected in the microbiology Laboratory of the Saint George Hospital in Beirut, mostly from ICU patients. In this report, molecular typing of isolates revealed one major PFGE pattern suggesting a clonal spread of this bacterium in the clinical setting [14]. A. baumannii in Lebanon was mostly implicated in ventilator associated pneumoniae $[15,16]$, urinary tract, intra-abdominal, blood and wound infections [17-19]. Furthermore, unique cases of necrotizing fasciitis caused by multi-drug resistant $A$. baumannii were also reported [20,21]. In one of the aforementioned necrotizing fasciitis cases, the strain was only sensitive to amikacin and polymyxin, challenging therefore the available treatment [21]. Besides A. baumannii, other species in this genus such as $A$. junii and $A$. lwoffii were also reported in the Lebanese clinical settings [17]. Kanj et al. associated Acinetobacter anitratus bacteremia to an early relapse of thrombotic thrombocytopenic purpura in a 58 years old male during plasma exchange therapy in 2003 [22]. On the other hand, A. pittii was detected in the urine culture of a four-month-old child as well as from febrile gastroenteritis infected patient [23].

Apparently, the aforementioned studies showed that A. baumannii strains are highly prevalent, but endemic in the Lebanese clinical settings. Several issues could be raised here: what about the co-isolation of other Gram-negative bacilli from the site of infection such as $P$. aeruginosa and $E$. coli? Is the threat attributed to $A$. baumannii over-estimated in view of the virulence and 
pathogenicity of this genus? What about nonbaumannii Acinetobacter species? Are identification procedures to the species level reliable? All this raises a big question mark about the real epidemiology of this organism in Lebanon. For instance, in their study, Kanafani et al. showed that among the most common organisms causing ventilator associated pneumonia were $A$. anitratus It is the same as A. baumannii but also Pseudomonas aeruginosa; the difference between the number of isolates was not significant (11 vs 8 isolates, respectively) [16]. Similarly, Awad et al., reported close prevalence of $P$. aeruginosa (31\%) and $A$. baumannii (37\%) isolated from ventilator associated pneumoniae affected patients in the intensive care unit [15]. In another study, Acinetobacter spp along with Candida and Escherichia coli predominated in catheter associated urinary tract infections [18]. In intraabdominal and urinary tract infections, Acinetobacter spp was not among the most common causative agents [19]. Indeed, the prevalence of this bacterium was comparable to $P$. aeruginosa [19]. Moreover, Chamoun et al. conducted a nationwide study where microbiological data were retrieved from 16 different hospitals across Lebanon from January 2011 till December 2013. This study showed that the most common Gram-negative bacillus causing nosocomial infections was $E$. coli followed by $P$. aeruginosa and Klebsiella spp [24], showing a secondary involvement of A. baumannii.

As for clinical outcomes, Jurdak et al. conducted a study where the mortality rate was assessed in patients with nosocomial pneumoniae admitted to the intensive care unit in a tertiary care center in Lebanon. Statistical analysis showed that the only significant differences between survivals and deceased were the duration of mechanical ventilation and the patient's age [25]. Another study conducted at the American University of Beirut Medical Center showed that multi-drug resistant A. baumannii infections resulted in a high mortality rate among ICU patients ranging from $52 \%$ to $66 \%$ between 2007 and 2014. Although not statistically significant, the complications due to A. baumannii infections were more correlated with the resistant strains than the susceptible ones, except for the acute respiratory distress syndrome. Despite all this, the attributable mortality rates could not have been calculated since these latter were more likely seen in older patients i.e. those with invasive device use, trauma, multiple comorbidities and cancer [17]. One study, addressing patients with neutropenic fever have found that increased in-hospital mortality rate was associated with the duration of neutropenia and treatment modification for clinical deterioration [26]. Moreover, in concordance with previous worldwide studies, Ballouz et al. found that prolonged hospital stay that is often associated with a loss of functionality, as well as antibiotic and steroid exposure especially in critically ill patients, increases the risk of exhibiting A. baumannii bacteremia with poor outcome [27]. Dahdouh et al. conducted a study on ninetyfive $A$. baumannii strains isolated from a tertiary care center in Beirut and showed that their virulence profiles were highly variable and without a specific pattern [28]. In addition, no specific association was observed between resistance patterns in these isolates and their virulence profiles. The relationship between different virulence factors including resistance is highly complex in A. baumannii species and requires more investigations [28]. All of this might suggest that the pathogenicity attributed to $A$. baumannii might have been over-estimated over the past years.

Identifying Acinetobacter to the specie level is another concern in the Lebanese hospitals. Indeed, the majority of the clinical laboratories perform biochemical identification. This methodology has poor specificity and cannot differentiate between different Acinetobacter species including A. baumannii, A. pittii, A. nosocomialis and A. calcoaceticus [6]. This is mainly due to their close phenotypic and genetic relatedness [6]. Non baumannii species differ from A. baumannii in terms of antibiotic susceptibility [29]. Lee et al. found that non baumannii strains were more resistant to colistin than their counterparts; for instance, Acinetobacter genomospecies 14TU are shown to have natural resistance to colistin [29]. One study conducted in Japan, used MALDI-TOF for the identification of Acinetobacter spp isolated from blood cultures. In this study, 13 different species of Acinetobacter were identified. Surprisingly, the incidence of $A$. pittii exceeded that of $A$. baumannii $(34.1 \%$ vs $17.9 \%$ respectively) [30]. In view of this, the accurate identification of Acinetobacter spp is instrumental for the appropriate management of the infection and for a more rational judgement of the real threat imposed by baumannii and non baumannii Acinetobacter as per the prevalence, clinical outcome and level of antibiotic resistance.

\section{Epidemiology of carbapenem resistant Acinetobacter species in Lebanon}

Humans

As mentioned earlier, Acinetobacter species are naturally resistant to a variety of clinically important antimicrobial agents. In addition, they have acquired 
complex mechanisms of resistance making them resistant to the last resort therapeutic agents available such as carbapenems and colistin. In this context, epidemiological studies, chasing the evolution and dissemination of multi-drug resistant GNB including Acinetobacter spp are warranted for appropriate empirical treatment and for the improvement of infection control measures. Multi-locus sequence typing (MLST) and Pulse field gel electrophoresis (PFGE) are the most common molecular tools used for the typing and tracking of multi-drug resistant organisms [6].

In Lebanon, resistance to extended spectrum cephalosporins in A. baumannii has been observed since early 1980s with the first report of Acinetobacter spp showing a significant increase in a tertiary care center in Beirut following hostilities' outbreak [13]. During the same decade, the isolation of seven hundred thirteen Acinetobacter spp over a seven-years period at Makassed General Hospital was reported. The majority of these isolates were multi-drug resistant, including resistance to third generation cephalosporins and aminoglycosides; carbapenems became therefore the treatment of choice for these infections [31]. Few years later, carbapenem resistant Acinetobacter species started to emerge in this same hospital [32] as well as other Lebanese hospitals [12-14,33]. Hamouche et al. reported the drop of imipenem susceptibility from $95.3 \%$ in 2006 to $44.6 \%$ in 2009 in A. baumannii clinical isolates [34]. A study conducted at a major tertiary care center in Beirut showed that the majority (82/90) of $A$. baumannii isolated from patients with bacteremia were resistant to carbapenem but remained susceptible to tigecycline and colistin [27]. In this study, colistin susceptibility was tested by the KirbyBauer technique [27]; this technique is not considered accurate for testing the susceptibility to colistin. On the contrary, MIC determination should be implemented in order to correctly assess the level of colistin resistance [35]. Another study done by Awad et al. reported the detection of carbapenem resistant and colistin/tigecycline susceptible $A$. baumannii strains isolated from patients affected by ventilator associated pneumoniae [15].

In 2008, Zarrilli et al. described the mechanism of carbapenem resistance in A. baumannii isolated from Saint George hospital in Beirut. In fact, resistance was mediated by a plasmid encoded OXA-58 gene flanked by IS $A b a 3$ and IS 18 elements. The strains belonged to one major PFGE pattern and one sequence type "ST2" [36]. In the following years, OXA-23 genes became predominant in $A$. baumannii strains isolated from the
Lebanese hospitals [5]. The PFGE type A'/ST2 prevailed in North Lebanon as well as in Beyrouth suggesting a clonal dissemination of this cluster across the country [5]. In 2011, Hammoudi et al. reported the detection of A. baumannii co-harboring OXA-23 and GES-11 beta lactamase genes [10]. The co-existence of OXA-23 and GES-11 was further described in $A$. baumannii isolated in nine Lebanese hospitals in 2012 where the insertion sequence ISAbal was detected upstream of the OXA-23 gene in hundred percent of the strains [37]. Furthermore, eighteen different pulsotypes were detected with the pulsotype 17 being predominant in many Lebanese hospitals revealing, again, a clonal dissemination of Acinetobacter across the country. On the other hand, the variety of pulsotypes suggests a horizontal transfer of resistance genes between the different clones [37]. At Saint George Hospital-UMC located in Beirut, Dahdouh et al. reported the predominance of OXA-23 producing A. baumannii strains belonging to the international clone ICII [28]. In this study, carbapenem resistance mediated by the blaOXA-23 gene was positively associated with ICII [28]. It is worth mentioning that in this study, three $A$. haemolyticus, one Acinetobacter radioresistens/ lwoffii and one Acinetobacter junii/johnsonii were also reported [28]. Other beta lactamase encoding genes detected in clinical isolates of $A$. baumannii included OXA-24 and hyperproduced ADC genes [7,38]. Similarly, non baumannii strains of Acinetobacter were detected by Hajjar et al. but were not explored further [7]. A study conducted at the American University of Beirut Medical center (AUBMC), a major tertiary care center located in Beirut, revealed an increase of OXA23 positive $A$. baumannii strains between two outbreaks, the first one described in 2007/2008 and the second one in 2013 [39]. Interestingly, strains isolated in both periods revealed only $22 \%$ of genomic relatedness. This suggests on one hand the rapidly changing diversity of this species, and on the other hand the significance of horizontal gene transfer between different clusters over the six years interval [39]. In view of its importance at the national level as a major medical center receiving patients from all over the country, the AUBMC results could be considered as representative of the country.

From a different perspective, the Syrian crisis imposed a socio-economic burden that might have an influence on health and disease including infectious diseases and bacterial resistance in Lebanon. In 2012, Rafei et al. reported the first detection of four NDM-1 producing A. baumannii strains isolated from Syrian refugees in Tripoli, North of Lebanon. PFGE and 
MLST analysis showed that the strains formed one PFGE cluster and belonged to the ST85 [40]. NDM-1 gene was previously described in Lebanon in clinical strains of Klebsiella pneumoniae in 2010 isolated from Iraqi patients but not in Acinetobacter species [40]. NDM-1 positive ST85 A. baumannii clinical isolates were later detected also in Syrian refugees but also in Lebanese patients within the same city [11]. Thereafter, NDM-1 producing A. baumannii were isolated from other Lebanese patients, however these latter belonged to a different sequence type "ST25" [41]. Noteworthy, in their study, Rafei et al. found that carbapenem resistance was significantly more prevailing among Syrian refugees than Lebanese patients [41]. Salloum et al. conducted a genomic analysis on two NDM-1 producing $A$. baumannii isolated in the aforementioned studies and found that NDM-1 was located on a nonconjugative plasmid [42]. The micro-diversity identified between the two isolates emphasizes the role of refugees in reshaping the diversity of multi-drug resistant organisms in Lebanon [42]. As for non- $A$. baumannii strains, a single study reported the first detection of $A$. pittii harboring the blaNDM-1 or the blaOXA-72 gene from a hospital located in the north of Lebanon [23]. This study highlights the importance of non baumannii strains in the clinical settings as suggested by other worldwide studies.

\section{Animals and environment}

Nowadays, it is widely accepted that multi-drug resistant organisms are no more confined to the hospital settings; rather they are disseminated in animals and environment [1]. A countrywide study conducted in $2012 / 2013$ by Rafei et al. in Lebanon reported that Acinetobacter species isolated from animals are mainly susceptible to the majority of antibiotics tested and lacked carbapenemase encoding genes [8]. Furthermore, these strains belonged to 36 different STs; twentyfour were of new sequence types [8]. In this study, only one $A$. baumannii isolated from a horse's oral cavity and one $A$. pittii isolated from a rabbit oral cavity were harboring the OXA-143 and OXA-24 genes respectively [8]. Furthermore, OXA-23 with/without OXA-58 carbapenemase encoding gene, were detected in A. baumannii strains of livestock origin [43]. Transconjugation experiments suggested that OXA-23 was chromosomally encoded while OXA-58 was plasmid mediated [43]. Furthermore, MLST analysis revealed that isolated strains were of novel sequence types except for ST20 and ST2. ST2 was reported in Lebanon as well as in different outbreaks worldwide [43]. More recently, A. baumannii harboring the ADC gene was detected in a fecal sample of poultry origin in the south Lebanon [1] and A. calcoaceticus producing the OXA-72 gene was described in vegetables purchased from a local market in Beirut $[4,44]$. The presence of multi-drug resistant organisms in vegetables could be the result of direct animal contamination or indirect environmental contamination with soil or irrigation water [44].

Countrywide studies in Lebanon showed that Acinetobacter species isolated from water and soil samples were susceptible to the majority of antibiotics including cephalosporins, carbapenem and non-beta lactam antibiotics $[4,8]$. Despite the absence of bacterial resistance, the noticeable findings in these studies is the predominance of non baumannii Acinetobacter species such as: A. pittii, A. calcoaceticus, Acinetobacter guillouiae and Acinetobacter bereziniae [4,8].

Overall, the dissemination of resistant Acinetobacter spp in Lebanon appears to be polyclonal and mediated primarily by the diffusion of resistance determinants namely blaOXA-23 in the clinical settings and livestock. Environmentally speaking, although isolated strains are susceptible to the most common antibiotics used in the human medicine; the detection of Acinetobacter species with novel sequence types in the environment is of particular importance. These strains with negligible knowledge on their intrinsic resistance, level of pathogenicity, and virulence might be transmitted to humans and animals, acquire resistance determinants, and cause infections of unknown consequences [30]. In the one health concept, this emphasizes that clinical settings, animals, and the surrounding environment form an interconnected chain. The successful control of the spread of bacterial resistance needs to be addressed though national interventions at all levels.

\section{Acinetobacter spread in Lebanon, what needs to be done?}

The evolution of resistance and the continuous emergence of new genetic determinants occurs naturally in bacteria [45]. Being intrinsically mediated, this phenomenon cannot be stopped but rather should be slowed down and controlled. Antibiotic stewardship programs aiming to control the misuse and over use of antibiotics in hospitals, community and animals are meant to make this happen. On the other hand, infection control measures that prevent the wide dissemination of multi-drug resistant organisms at the human, animal and environmental interface are also warranted. In Lebanon, the implementation of these measures is challenging due to the difficulty of hospital staff 
cohorting, especially in staff shortage situations which warrant absolute cooperation from the hospital and nursing administration. Furthermore, patient's isolation imposes psychological well-being and hospital financial costs [46]. Unfortunately, it looks like, infection control measures (if applied) come after the situation has become endemic as it is the case of several hospitals in Beirut and North Lebanon [47].

Regardless of the aforementioned obstacles that face the implementation of preventative measures; these latter are warranted due to the wide dissemination of multi-drug resistant organisms in many ecosystems in Lebanon. According to Kanafani et al., two weeks of additional hospitalization might result from a single central line-associated bloodstream infection or ventilator associated pneumonia episode due to multidrug resistant Acinetobacter species [17]. Knowing that the average cost of one day ICU stay is $\$ 1750$ [17]. Preventative measures should target equipment/environmental disinfection, hand hygiene program for health care workers, MDRO's screening upon admission and patients' isolations [11]. These latter should be practiced even if in a given hospital, the prevalence of a MDROs is still low. An example of a successful containment of an A. baumannii outbreak in Lebanon is at the AUBMC [39]. In this case, onadmission and weekly screening of all respiratory, ICU and high risk patients was performed. The screening included oropharyngeal, umbilical, axillary, perianal and rectal swabbing. Upon the screening results, contact isolation is taken for all infected/colonized patients until discharge. Furthermore, on a daily basis, these patients are bathed with chlorhexidine. This led to the containment of the outbreak in 2013 as compared to 2007-2008 [39].

Another important action to be taken in the country is to upgrade the medical labs and empower the microbiology sections. Clinical microbiologists are the first to encounter and track the evolution and the emergence of new resistance determinants. The fast and correct tracking of bacterial resistance helps on one hand in guiding physicians to the appropriate antibiotic empirical treatment, and on the other hand in alerting the infection control personnel. The clinical microbiology staff should be therefore trained to correctly and appropriately detect and report resistance of a given bacterium to an antibiotic (intrinsic versus acquired resistance) as well as to determine the phenotypic and genotypic mechanism of resistance.

Furthermore, an appropriate and reliable methodology of Acinetobacter identification is also needed. This might be expensive for individual hospitals, however, the establishment of a national referee lab for Acinetobacter can solve the issue. Such a laboratory can benefit from advanced technology i.e. Matrix-assisted laser desorption/ionization time-offlight mass spectrometry (MALDI-TOF MS). This technique is increasingly used for the reliable identification of microorganisms including Gram positive and Gram-negative bacteria [30]. MALDITOF is a cost-effective tool, allowing the fast and simple bacterial identification using a small amount of sample [29]. The monitoring of colistin resistance using MIC determination is also warranted in view of the worldwide emergence and dissemination of colistin resistance in all ecosystems [1].

Figure 1. Emergence of resistance in Acinetobacter spp isolated from humans, animals and environment in Lebanon based on the bacterial isolation date.

Phenotypes of resistance

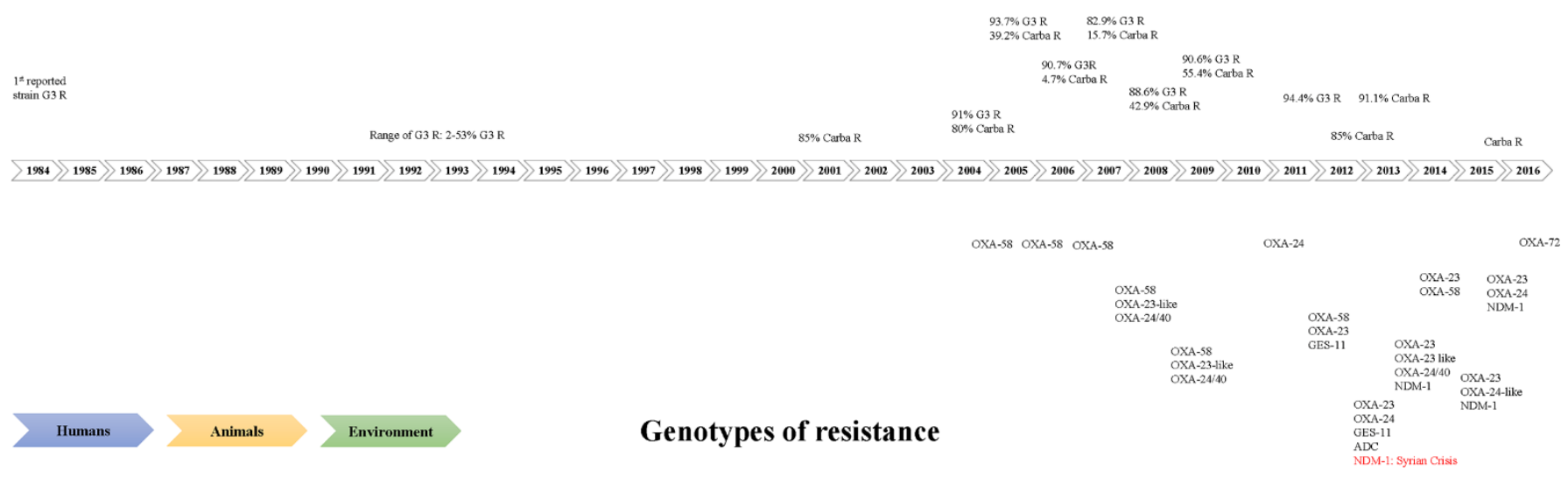

$\mathrm{G} 3=$ third generation cephalosporins, carba $=$ carbapenems, $\mathrm{R}=$ resistant. 


\section{Conclusion}

The dissemination of multi-drug resistant Acinetobacter species is a public health concern that needs to be carefully addressed in Lebanon. Although problematic and controversial, the clinical outcome of A. baumannii infections are mostly due to the severity of the underlying illness rather than the pathogenicity of the bacterium. More investigations on the risk factors responsible for the acquisition of MDR Acinetobacter species and other MDROs in hospitals is therefore warranted. In addition, studies on how to overcome the challenges of implementing antibiotic stewardship and infection control programs are urgently needed. As shown in Figure 1, the big majority of studies in Lebanon addressed the epidemiology of Acinetobacter spp in humans; while the investigation of animals and the environment remains very scarce. In the one health concept, this review emphasizes the need of a multilevel cooperative efforts in human, animals and environment in attempt to contain the wide dissemination of MDROs in Lebanon.

\section{References}

1. Dandachi I, Sokhn ES, Dahdouh EA, Azar E, El-Bazzal B, Rolain JM, Daoud Z (2018) Prevalence and characterization of multi-drug-resistant gram-negative bacilli isolated from Lebanese poultry: A nationwide study. Front Microbiol 9: 550.

2. Asif M, Alvi IA, Rehman SU (2018) Insight into acinetobacter baumannii: Pathogenesis, global resistance, mechanisms of resistance, treatment options, and alternative modalities. Infect Drug Resist 11: 1249-1260.

3. Bravo Z, Chapartegui-Gonzalez I, Lazaro-Diez M, RamosVivas J (2018) Acinetobacter pittii biofilm formation on inanimate surfaces after long-term desiccation. J Hosp Infect 98: 74-82.

4. Al Atrouni A, Hamze M, Rafei R, Eveillard M, Joly-Guillou ML, Kempf M (2016) Diversity of acinetobacter species isolated from different environments in Lebanon: A nationwide study. Future Microbiol 11: 1147-1156.

5. Rafei R, Dabboussi F, Hamze M, Eveillard M, Lemarie C, Gaultier MP, Mallat H, Moghnieh R, Husni-Samaha R, JolyGuillou ML, Kempf M (2014) Molecular analysis of acinetobacter baumannii strains isolated in Lebanon using four different typing methods. PLoS One 9: e115969.

6. Jamal S, Al Atrouni A, Rafei R, Dabboussi F, Hamze M, Osman M (2018) Molecular mechanisms of antimicrobial resistance in acinetobacter baumannii, with a special focus on its epidemiology in Lebanon. J Glob Antimicrob Resist 15: 154-163.

7. Hajjar Soudeiha M, Dahdouh E, Daoud Z, Sarkis DK (2018) Phenotypic and genotypic detection of beta-lactamases in acinetobacter spp isolates recovered from Lebanese patients over a 1-year period. J Glob Antimicrob Resist 12: 107-112.

8. Rafei R, Hamze M, Pailhories H, Eveillard M, Marsollier L, Joly-Guillou ML, Dabboussi F, Kempf M (2015) Extrahuman epidemiology of acinetobacter baumannii in Lebanon. Appl Environ Microbiol 81: 2359-2367.

9. Wong D, Nielsen TB, Bonomo RA, Pantapalangkoor P, Luna B, Spellberg B (2017) Clinical and pathophysiological overview of acinetobacter infections: A century of challenges. Clin Microbiol Rev30: 409-447.

10. Hammoudi D, Moubareck CA, Kanso A, Nordmann P, Sarkis DK (2015) Surveillance of Carbapenem non susceptible gram negative strains and characterization of Carbapenemases of classes, A, B and D in a Lebanese hospital.. J Med Liban 63: 66-73.

11. Al Atrouni A, Hamze M, Jisr T, Lemarie C, Eveillard M, JolyGuillou ML, Kempf M (2016) Wide spread of OXA-23producing carbapenem-resistant acinetobacter baumannii belonging to clonal complex II in different hospitals in Lebanon. Int J Infect Dis52: 29-36.

12. Moghnieh RA, Abdallah DI, Fawaz IA, Hamandi T, Kassem M, El-Rajab N, Jisr T, Mugharbil A, Droubi N, Al Tabah S, Sinno L, Ziade F, Daoud Z, Ibrahim A (2017) Prescription patterns for tigecycline in severely ill patients for non-FDA approved indications in a developing country: A compromised outcome. Front Microbiol 8: 497.

13. Matar GM, Gay E, Cooksey RC, Elliott JA, Heneine WM, Uwaydah MM, Matossian RM, Tenover FC (1992). Identification of an epidemic strain of acinetobacter baumannii using electrophoretic typing methods. Eur J Epidemiol 8: 9-14.

14. Di Popolo A, Khan AU, Daoud Z, Bagattini M, Afif C, Triassi M, Hakimé NI, Zarrilli R (2007) Epidemiology and mechanism of resistance of an outbreak of multidrug-resistant acinetobacter baumannii at in a Lebanese hospital. Int $\mathrm{J}$ of Antimicrob Agents 29 Suppl 2: 269.

15. Awad LS, Abdallah DI, Mugharbil AM, Jisr TH, Droubi NS, El-Rajab NA, Moghnieh RA (2017) An antibiotic stewardship exercise in the ICU: Building a treatment algorithm for the management of ventilator-associated pneumonia based on local epidemiology and the 2016 infectious diseases society of America/American thoracic society guidelines. Infect Drug Resist 11: 17-28.

16. Kanafani ZA, Kara L, Hayek S, Kanj SS (2003) Ventilatorassociated pneumonia at a tertiary-care center in a developing country: Incidence, microbiology, and susceptibility patterns of isolated microorganisms. Infect Control Hosp Epidemiol 24: 864-869.

17. Kanafani ZA, Zahreddine N, Tayyar R, Sfeir J, Araj GF, Matar GM, Kanj SS (2018) Multi-drug resistant acinetobacter species: A seven-year experience from a tertiary care center in Lebanon.Antimicrob Resist Infect Control 7: 1-8.

18. Kanj SS, Zahreddine N, Rosenthal VD, Alamuddin L, Kanafani Z, Molaeb B (2013) Impact of a multidimensional infection control approach on catheter-associated urinary tract infection rates in an adult intensive care unit in Lebanon: International nosocomial infection control consortium (INICC) findings. Int J Infect Dis 17: e686-690.

19. Hayajneh WA, Hajj A, Hulliel F, Sarkis DK, Irani-Hakimeh N, Kazan L, Badal RE (2015) Susceptibility trends and molecular characterization of gram-negative bacilli associated with urinary tract and intra-abdominal infections in Jordan and Lebanon: SMART 2011-2013. Int J Infect Dis 35: 56-61.

20. Nehme A, Joukhadar NI, Saidy E, Darwiche M, Aouad DK, Abdel Nour HG (2018) Fatal acinetobacter baumanii necrotizing fasciitis following open reduction internal fixation in a polytrauma patient. Case Rep Infect Dis 2018: 1-5.

21. Sinha N, Niazi M, Lvovsky D (2014) A fatal case of multidrug resistant acinetobacter necrotizing fasciitis: The changing scary face of nosocomial infection. Case Rep Infect Dis 705279.

22. Kanj NA, Mikati AR, Kfoury Baz EM (2003) Early relapse of thrombotic thrombocytopenic purpura during therapeutic 
plasma exchange associated with acinetobacter anitratus bacteremia. Ther Apher Dial 7: 119-121.

23. Al Atrouni A, Joly-Guillou ML, Hamze M, Kempf M (2016) Emergence of NDM-1 and OXA-72 producing Acinetobacter pittii clinical isolates in Lebanon. New Microbes New Infect 22: 43-44.

24. Chamoun K, Farah M, Araj G, Daoud Z, Moghnieh R, Salameh P, Saade D, Mokhbat J, Abboud E, Hamze M, Abboud E, Jisr T, Haddad A, Feghali R, Azar N, El-Zaatari M, Chedid M, Haddad C, Zouain Dib Nehme M, Barakat A, Husni R (2016) Surveillance of antimicrobial resistance in Lebanese hospitals: Retrospective nationwide compiled data. Int J Infect Dis 46: 64-70.

25. Jurdak H, Moghnieh R, Lababidi HM (2006) Nosocomial pneumonia due to Acinetobacter Baumanii in the intensive care unit. Chest 130, 4, Supplement: 262.

26. Kanafani ZA, Dakdouki GK, El-Chammas KI, Eid S, Araj GF, Kanj SS (2007) Bloodstream infections in febrile neutropenic patients at a tertiary care center in Lebanon: A view of the past decade. Int J Infect Dis 11: 450-453.

27. Ballouz T, Aridi J, Afif C, Irani J, Lakis C, Nasreddine R, Azar E (2017) Risk factors, clinical presentation, and outcome of acinetobacter baumannii bacteremia. Front Cell Infect Microbiol 7: 156

28. Dahdouh E, Hajjar M, Suarez M, Daoud Z (2016) Acinetobacter baumannii isolated from Lebanese patients: Phenotypes and genotypes of resistance, clonality, and determinants of pathogenicity. Front Cell Infect Microbiol 6: 163.

29. Jeong S, Hong JS, Kim JO, Kim KH, Lee W, Bae IK, Lee K, Jeong SH (2016) Identification of acinetobacter species using matrix-assisted laser desorption ionization-time of flight mass spectrometry. Ann Lab Med 36: 325-334.

30. Kishii K, Kikuchi K, Matsuda N, Yoshida A, Okuzumi K, Uetera Y, Yasuhara H, Moriya K (2014) Evaluation of matrixassisted laser desorption ionization-time of flight mass spectrometry for species identification of acinetobacter strains isolated from blood cultures. Clin Microbiol Infect20: 424-430.

31. Shaar TJ, Al-Hajjar R (2000) Antimicrobial susceptibility patterns of bacteria at the makassed general hospital in Lebanon. Int J Antimicrob Agents 14: 161-164.

32. Daher JA, Shaar TJ, Lababidi HM (2006) Microorganisms profile and antimicrobial susceptibility in the intensive care unit: The emergence of a resistant pathogen Acinetobacter Baumanii. Chest 2006 130, 4, Supplement: 217.

33. Araj GF, Avedissian AZ, Ayyash NS, Bey HA, El Asmar RG, Hammoud RZ, Itani LY, Malak MR, Sabai SA (2012) A reflection on bacterial resistance to antimicrobial agents at a major tertiary care center in Lebanon over a decade. J Med Liban 60: 125-135.

34. Hamouche E, Sarkis DK (2012) Evolution of susceptibility to antibiotics of escherichia coli, klebsiella pneumoniae, pseudomonas aeruginosa and acinetobacter baumanii, in a university hospital center of beirut between 2005 and 2009 . Pathol Biol 60: e15-20.

35. Dandachi I, Leangapichart T, Daoud Z, Rolain JM (2018) First detection of $m c r-1$ plasmid mediated colistin resistant E.coli in Lebanese poultry. J Glob Antimicrob Resist 12: 137-138

36. Zarrilli R, Vitale D, Di Popolo A, Bagattini M, Daoud Z, Khan AU, Afif C, Triassi M (2008) A plasmid-borne blaOXA-58 gene confers imipenem resistance to acinetobacter baumannii isolates from a Lebanese hospital. Antimicrob Agents Chemother52: 4115-4120.

37. Hammoudi D, Moubareck CA, Hakime N, Houmani M, Barakat A, Najjar Z, Suleiman M, Fayad N, Sarraf R, Sarkis
DK (2015) Spread of imipenem-resistant acinetobacter baumannii co-expressing OXA-23 and GES-11 carbapenemases in Lebanon. Int J Infect Dis 36: 56-61.

38. Hammoudi Halat D, Moubareck CA, Sarkis DK (2017) Heterogeneity of carbapenem resistance mechanisms among gram-negative pathogens in Lebanon: Results of the first crosssectional countrywide study. Microb Drug Resist 23: 733-743.

39. Kanj SS, Tayyar R, Shehab M, El-Hafi B, Rasheed SS, Kissoyan KAB, Kanafani ZA, Wakim RH, Kara Zahreddine N, Araj GF, Dbaibo G, Matar GM (2018) Increased blaOXA-23like prevalence in Acinetobacter baumannii at a tertiary care center in Lebanon (2007-2013). J Infect Dev Ctries 12: 228234. doi: $10.3855 /$ jidc. 9642

40. Rafei R, Dabboussi F, Hamze M, Eveillard M, Lemarie C, Mallat H, Rolain JM, Joly-Guillou ML, Kempf M (2014) First report of blaNDM-1-producing acinetobacter baumannii isolated in Lebanon from civilians wounded during the syrian war. Int J Infect Dis 21: 21-23.

41. Rafei R, Pailhories H, Hamze M, Eveillard M, Mallat H, Dabboussi F, Joly-Guillou ML, Kempf M (2015) Molecular epidemiology of acinetobacter baumannii in different hospitals in tripoli, Lebanon using bla OXA-51-like sequence based typing. BMC Microbiol 15: 103.

42. Salloum T, Tannous E, Alousi S, Arabaghian H, Rafei R, Hamze M, Tokajian S (2018) Genomic mapping of ST85 blaNDM-1 and blaOXA-94 producing acinetobacter baumannii isolates from syrian civil war victims. Int J Infect Dis 74: 100-108.

43. Al Bayssari C, Dabboussi F, Hamze M, Rolain JM (2015) Emergence of carbapenemase-producing pseudomonas aeruginosa and acinetobacter baumannii in livestock animals in Lebanon. J Antimicrob Chemother 70: 950-951.

44. Al Atrouni A, Kempf M, Eveillard M, Rafei R, Hamze M, JolyGuillou ML (2015) First report of oxa-72-producing acinetobacter calcoaceticus in Lebanon. New Microbes New Infect 9: 11-12.

45. Blazquez J, Oliver A, Gomez-Gomez JM (2002) Mutation and evolution of antibiotic resistance: Antibiotics as promoters of antibiotic resistance? Curr Drug Targets 3: 345-349.

46. Moghnieh R, Siblani L, Ghadban D, El Mchad H, Zeineddine R, Abdallah D, Ziade F, Sinno L, Kiwan O, Kerbaj F, El Imad Z (2016) Extensively drug-resistant acinetobacter baumannii in a Lebanese intensive care unit: Risk factors for acquisition and determination of a colonization score. J Hosp Infect 92: 4753.

47. Hammoudi D, Ayoub Moubareck C, Aires J, Adaime A, Barakat A, Fayad N, Hakime N, Houmani M, Itani T, Najjar Z, Suleiman M, Sarraf R, Karam Sarkis D (2014) Countrywide spread of OXA-48 carbapenemase in Lebanon: Surveillance and genetic characterization of carbapenem-non-susceptible enterobacteriaceae in 10 hospitals over a one-year period. Int $\mathrm{J}$ Infect Dis 29: 139-144.

\section{Corresponding author}

Ziad Daoud, PhD, Professor

Clinical Microbiology

Faculty of Medicine and Medical Sciences

University of Balamand www.balamand.edu.lb

POBox: 33, Amioun, Lebanon

Tel: +961.6.930250 (ext 3819)

Fax: +961.6.930250 (ext 3819)

Email : ziad.daoud@balamand.edu.lb

Conflict of interests: No conflict of interests is declared. 